\section{A computer data base system for indexing research papers}

\section{ROBERT A. GLASS, LARRY J. KAETZEL, and GEORGE R. SMITH}

National Bureau of Standards, National Engineering Laboratory Center for Building Technology, Washington, D.C. 20234

A set of computer programs has been developed that allows the cataloging, classifying, retrieval, and editing of citations of research papers through the use of a computer terminal. The system is designed to help the user organize, edit, file, and retrieve copies of research papers.

The program allows the user to list alphabetically (by first author) all references stored and retrieve them through the computer terminal or hard-copy printing device. A major advantage of implementing a system for personal copies of research papers is that it allows the user to consider the references as forming a research data base and allows the user to search through them using new search strategies. The key-word mode allows the user to select up to six key words (up to 16 characters each). The key words are then extracted whenever they appear in either the key-word index or the title of the research paper. The extracted record is then printed (hard copy) or appears on the computer display terminal (at the option of the user). The record appearing lists the author, record number, file code, publication date, title, publication name, volume and page number, and up to six key words.

\section{Input}

The user makes decisions concerning the file arrangement and the key words for each reference article and enters each reference sequentially. Later, changes can be made through the computer text editor. Once data have been entered, the user can search and retrieve any article through a key-word or author search.

\section{Modes of Operation}

Data Base Supervisor Program. When the data base system is activated, a message is sent to the user terminal requesting the data base name. At this time, the operator should type "File name." By doing so, the proper data base is assigned and data will be generated in the proper format for subsequent processing.

EDIT Mode. The user may edit the KGS data base by typing the command "EDIT." Execution of the EDIT mode begins by requesting one of the five EDIT modes: (1) search, (2) update, (3) append, (4) delete, and (5) return. All editing is performed by referencing the data base record number. Records are numbered from one to the last record in the file. There is no maximum number of records, except that which is dictated by disk storage capacity. Each data base record consists of 15 lines (elements). Maximum line (element) sizes are preassigned for each record.

SEARCH Mode. The SEARCH mode is used to locate a data base record. The program asks for an author's name and searches the data base for that author. When a record containing a match on the author's name is found, the number of that record is displayed along with the author and publication title. The program then asks if the search process should continue for that author. A "no" response terminates the SEARCH mode; a "yes" response continues the search until the end of the data base is reached.

UPDATE Mode. The UPDATE mode is used to modify an element or elements in a data base record that are already in the data base. The program first asks for the record number and then displays the 15 lines of text currently stored in the record. In addition to the text, the line or element number is affixed at the beginning of the line. These numbers, from 1 to 15 , are then used to identify the element.

By typing the line number, the program displays the current contents and asks for the updated information. The new text entered is then used to replace the old text. Replacement is by line only; no character string capability is available. The program then asks for the next line number to be updated. When updating is completed, an asterisk is typed, at which time the entire record is displayed and the user is given the option to save the updated record by typing "Y" or "N." An "N" response will default to the previously recorded text. The program then asks for the next record to be updated. When update is completed, an asterisk is typed and another EDIT operation is requested.

APPEND Mode. The APPEND mode is used to insert new records into the data base. In the APPEND mode, each element is requested by displaying first the element name and then a prompt ${ }^{1}$ to indicate that input is necessary. The operator enters the appropriate information for the entire record. The information is then displayed, and the option to save or discard the information is given. A "YES" response results in the request for the next record. By typing an asterisk the user terminates the APPEND mode, and another mode can be requested.

DELETE Mode. The DELETE mode is used to delete a record from the data base. The program first asks for the record number to be deleted and then displays the author and title contained in the record. The message "DELETE OK?" is then displayed. A "YES" response deletes the record by marking it "DELETED." An asterisk returns for request for another EDIT mode.

RETURN Mode. By typing "RETURN," the user returns control to the data base supervisor program.

KEY WORD Mode. The KEY WORD mode is 
designed to search each data base record and produce a summary report of sorted key words found. The program is activated in the run mode by typing the word "KEYS." An option is also provided that allows output to be displayed on the user CRT or a hard-copy device. The report is generated in an alphanumeric sorted order. In addition to the list of key words, the number of occurrences is indicated. A summary is also presented that indicates the number of records in the data base, number of "deleted" records, and number of different key words.

SELECT DB Mode. The purpose of the SELECT DB mode is to allow the user to transfer control to another data base without terminating the KGS data base software package. This is useful if the user wishes to maintain publications on two or more areas of research. By typing "SELECT DB" the command "ENTER DATA BASE NAME" will be displayed. The data base name entered is subsequently used to activate the appropriate retrieval mode for data base manipulation. The valid data base names are maintained in the supervisor program and are modified by deleting or inserting as needs arise.

ONE KEY Mode. The purpose of the ONE KEY mode is to assist the data base operator in querying the data base for specific publication topics (key words). The user types a key word when requested, and the program searches the data base and displays a number of records (if any) that contained that key word. This is useful if a "snapshot" of the data base is desired, without displaying each data base record.

RETRIEVE Mode. Information retrieval and display of the KGS data base are performed using the RETRIEVE mode. Various search criteria such as key word or author name may be entered, and subsets of the data base may be extracted. Also included is the option for a field or character search of a data base record. A field search will attempt to match the characters entered by the operator with the characters contained in the data base element. A string search will search the entire element for a match of the characters entered by the operator, with any contiguous character match contained in the data base record element. The maximum number of characters in a string search is 100 . In the case in which the key word search option is employed, each of the six key-word fields plus the title field will be used for searching. Optional printer hard copy output is available. An option for the UNION or the INTERSECTION of more than one key word is also provided. In the "OR" condition, records must have all key words (maximum of six) in either the title or the key-word field. In the "AND" condition, a match on any of the entered key words will result in the record's being retrieved.

When a string search criterion is employed, the operator may enter the root of a key word, which will allow records to be retrieved that have that root plus variations. For example, entering characters "COL" might result in records having key words such as: color, colour, colors, colours.

\section{Operating Environment}

Language. The programs are written in FORTRAN V, Level 1, and may be used on a variety of computer systems. We are using a Perkin-Elmer ${ }^{3}$ Model 7/32 com. puter system. Program development and data base manipulation are performed using the Perkin-Elmer "MultiTerminal Manager" software package, which operates in a multitasking environment. Operator commands are performed using a cathode-ray tube (CRT) "dumb" terminal. Data base storage is maintained through the use of a Calcomp 25-million-character disk drive. Information retrieval is provided as an operator option, via the CRT command device or a medium-speed line printer.

Limitations. The retrieving and recording of a data base record are performed using subroutine "SYSIO." This program is made available through the PerkinElmer FORTRAN Run Time Library and provides a more efficient method of transferring the 372-character data base record between the computer's main memory and the peripheral disk storage device. A local run-time library manual should be consulted if this efficiency is desired. Otherwise, FORTRAN format statements may be substituted. The memory required for program execution is $23 \mathrm{~K}$ bytes for the main segment and $10 \mathrm{~K}$ bytes for library subroutine.

Availability. A listing of the data base supervisor program and the user-written subroutines and a description of a program parameters and retrieval formats are available free of charge from the National Bureau of Standards, National Engineering Laboratory, Center for Building Technology, Program Planning and Liaison Group, Washington, D.C. 20234. Request Tech. Note 1123.

\section{REFERENCE NOTE}

1. Kaetzel, L. J., Glass, R. A., \& Smith, G. M. A computer data base system for indexing research papers (NBS Tech. Note 1123), 1980.

\section{NOTES}

1. A prompt is a special character displayed by the computer to indicate that an operator command is expected.

2. Each element in the record is replaced with the characters "DELE."

3. The mention of the computer system referenced does not constitute any NBS endorsement of the product.

(Accepted for publication October 13, 1980.) 